\title{
Adverse Pregnancy Outcomes by Degree of Maternal Serum Analyte Elevation: A Retrospective Cohort Study
}

\author{
Amanda Yeaton-Massey, $\mathrm{MD}^{1}$ Rebecca J. Baer, MPH ${ }^{2,30}$ Larry Rand, MD ${ }^{3,4}$ \\ Laura L. Jelliffe-Pawlowski, $\mathrm{PhD}^{3,5}$ Deirdre J. Lyell, $\mathrm{MD}^{1}$
}

${ }^{1}$ Department of Obstetrics and Gynecology, Stanford University, Stanford, California

${ }^{2}$ Department of Pediatrics, University of California San Diego, La Jolla, California

${ }^{3}$ California Preterm Birth Initiative, University of California San

Francisco, San Francisco, California

${ }^{4}$ Department of Obstetrics, Gynecology, and Reproductive Sciences,

University of California San Francisco, San Francisco, California

${ }^{5}$ Department of Epidemiology and Biostatistics, University of

California San Francisco School of Medicine, San Francisco, California
Address for correspondence Amanda Yeaton-Massey, MD, Department of Obstetrics and Gynecology, Stanford University, 300 Pasteur Drive, G331, Stanford, CA 94305 (e-mail: amandaym@gmail.com).

Am J Perinatol Rep 2020;10:e369-e379.

\begin{abstract}
Keywords

- AFP

- hCG

- estriol

- inhibin

- adverse pregnancy outcomes

Objective The aim of this study was to evaluate rates of preterm birth (PTB) and obstetric complication with maternal serum analytes $>2.5$ multiples of the median (MoM) by degree of elevation.

Study Design Retrospective cohort study of singleton live-births participating in the California Prenatal Screening Program (2005-2011) examining PTB and obstetric complication for $\alpha$-fetoprotein (AFP), human chorionic gonadotropin (hCG), unconjugated estriol (uE3), and inhibin A (INH) by analyte subgroup ( 2.5 to $<6.0,6.0$ to $<10.0$, and $\geq 10.0$ MoM vs. $<2.5$ MoM).

Results The risk of obstetric complication increased with increasing hCG, AFP, and INH MoM, and were greatest for AFP and INH of 6.0 to $<10.0$ MoM. The greatest risk of any adverse outcome was seen for hCG MoM $\geq 10.0$, with relative risk (RR) of PTB $<34$ weeks of 40.8 (95\% confidence interval [Cl]: 21.7-77.0) and 13.8 (95\% Cl: 8.2-23.1) for obstetric complication.

Conclusions In euploid, structurally normal fetuses, all analyte elevations $>2.5 \mathrm{MoM}$ confer an increased risk of PTB and, except for uE3, obstetric complication, and risks for each are not uniformly linear. These data can help guide patient counseling and antenatal management.
\end{abstract}

Prenatal screening with maternal serum analytes is utilized to identify pregnancies at risk of aneuploidy and open neural tube defects. Elevated maternal serum analytes in the absence of aneuploidy and open neural tube defects have been associated with placental disorders, preterm birth (PTB) and obstetric complication such as preeclampsia, placental abruption, and small for gestational age (SGA). ${ }^{1-14}$

received

May 8, 2020

accepted

June 4,2020
DOI https://doi.org/

10.1055/s-0040-1716741. ISSN 2157-6998.
The body of existing literature on pregnancy outcomes with elevated maternal serum analytes tends to report outcome data for multiples of the median (MoM) $\geq 2.0$ or $\geq 2.5 \mathrm{MoM}$ (vs. those $<2.0 \mathrm{MoM}$ or $<2.5 \mathrm{MoM}$, respectively) ${ }^{1,9,15}$ or by centile (MoM $\geq 95$ th centile vs. $<95$ th centile). ${ }^{5,8}$ Outcomes have also been examined by subgroup of elevated analyte, up to $\geq 2.5$ MoM. ${ }^{3,16}$ We searched the
Copyright $\odot 2020$ by Thieme Medical Publishers, Inc., 333 Seventh Avenue, New York, NY 10001, USA. Tel: +1(212) 760-0888.
License terms

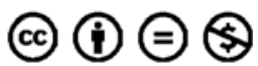


English language literature from 1950 to 2018 using PubMed and the search terms "elevated," "maternal," "analyte," " $\alpha$ fetoprotein," " $\alpha$-fetoprotein," "AFP," "human chorionic gonadotropin," "human chorionic gonadotrophin," "hCG," “estriol," "uE3," "inhibin," and "INH" and found that the highest maternal serum analyte elevations reported in conjunction with adverse pregnancy outcome for each second-trimester analyte were AFP $>5.0 \mathrm{MoM}^{12}$ hCG $>6.00 \mathrm{MoM}^{17}$ uE3 $>3.00,{ }^{7}$ and $\mathrm{INH}>2.5 \mathrm{MoM}^{16}$

The aim of our study was to describe rates of PTB and obstetric complication (composite variable, comprised of: preeclampsia, placental abruption, and SGA) among euploid, structurally normal fetuses with elevated maternal serum analytes by degree of analyte elevation. We hypothesized that the risk of PTB and obstetric complication would vary by the degree of maternal serum analyte elevation for women with analytes $>2.5$ MoM.

\section{Materials and Methods}

Our study sample $(n=1,464,139)$ was drawn from a live birth cohort database $(n=3,767,337)$ maintained by the California Office of Statewide Health Planning and Development for live births delivered between 2005 and 2011 (-Appendix 1). The birth cohort file contains detailed information on maternal and newborn characteristics derived from linked hospital discharge, birth certificate, and death records wherein all records for the mother and baby present from 1 year prior to the birth to 1 year after the birth are included. The file provides diagnoses and procedure codes based on the International Classification of Diseases, 9th Revision, Clinical Modification (ICD-9).

The sample was restricted to singletons linked to the California Prenatal Screening Program administered by the Genetic Disease Screening Program within the California Department of Public Health and included mothers who participated in second-trimester prenatal screening. Newborns with gestations at birth of $<20$ weeks or $>42$ weeks or birth weight three standard deviations above or below the mean for gestation and sex ${ }^{18}$ were excluded to correct for implausible estimates of gestational age or birth weight. Newborns with major structural anomalies (obtained from ICD-9 codes) or aneuploidy were also excluded. Anomalies were considered major if they were determined by clinical review to cause major morbidity and mortality identified during the birth hospitalization or resulting in hospitalization during the first year of life. ${ }^{19}$ Information about aneuploidy was gathered by the Genetic Disease Screening Program California Chromosomal Defect Registry personnel, who collect this information on all California births. ${ }^{20-23}$ Registry ascertainment sources included physicians, cytogenetic laboratories, hospitals, and prenatal diagnostic centers. All of these sources are mandated by California law to report diagnoses of aneuploidy to the Genetic Disease Screening Program.

Analytes in the second-trimester prenatal screen included serum AFP, hCG, uE3 (Perkin-Elmer Life Sciences, Waltham, MA), and, starting in July 2007, INH (Applied Biosystems,
Brea, CA). Serum was drawn between 15 and 20 weeks of gestation. Results were reported as MoM adjusted for gestational age, maternal weight, race or ethnicity, smoking status, and preexisting diabetes. ${ }^{24}$

Adverse pregnancy outcomes examined included PTB and obstetric complication. Obstetric complication was a composite variable including preeclampsia, placental abruption, and SGA. Gestational age at birth was obtained from birth certificate records (for births before 2007, last menstrual period was used; for births 2007 or later, best obstetric estimate was used). Subtypes of PTB were grouped as follows: premature rupture of membranes (PROM), spontaneous labor with intact membranes, and provider-initiated PTB. As previously described, all pregnancies with an indication of PROM in the newborn's birth certificate or mother's hospital discharge records were included in the PROM group. ${ }^{5}$ Women without an indication of PROM, but with indication of premature labor or tocolytic medication use in birth certificate or hospital discharge records, were included in the spontaneous labor with intact membranes group. Pregnancies were classified as having a provider-initiated PTB if they were without PROM, premature labor or tocolytic administration, if there was a code for "medical induction" or "artificial rupture of membranes," or if there was a cesarean delivery without any of the aforementioned codes. PTBs were also sub grouped by gestation $<34$ weeks.

Outcomes included in the obstetric complication composite (preeclampsia, placental abruption, and SGA) were determined from hospital discharge ICD-9 diagnostic codes as follows: preeclampsia/eclampsia (codes 642.4, 642.5, 642.6, or 642.7); placental abruption (codes 641.2, newborn code 762.1 ). SGA was defined as $<10$ th percentile for gestational age at delivery and sex. ${ }^{18}$

To determine analyte cut points, we generated receiver operator curves for each maternal serum analyte, comparing the rate of adverse pregnancy outcome (PTB $<37$ weeks and obstetric complication) for each analyte with $\mathrm{MoM}>2.5$. No clear inflection point was observed for any of the four analytes (AFP, hCG, uE3, INH). Given the lack of a clear cut point, we elected to examine the data categorically. We defined three clinically accessible categories a priori, using a MoM of $\geq 2.5$ as the beginning of "elevated." Our three categories of elevated analyte were MoM 2.5 to $<6.0,6.0$ to $<10.0$, and $\geq 10.0$. These three subgroups were compared with women with an analyte $\mathrm{MoM}<2.5$. For each analyte, we calculated both the risk of PTB and obstetric complication using logistic regression to calculate relative risks (RR) and 95\% confidence intervals (CI). All analyses were conducted using Statistical Analysis Software (SAS) version 9.4 (Cary, NC). The study was approved by the Committee for the Protection of Human Subjects within the Health and Human Services Agency of the State of California. Data from the California Prenatal Screening Program were obtained through the California Biobank Program (Screening Information System request no. 476). Data were obtained with an agreement that the California Department of Public Health is not responsible for the results or conclusions drawn by the authors of this publication. 
Table 1 Maternal demographic characteristics

\begin{tabular}{|c|c|}
\hline & $n(\%)$ \\
\hline \multicolumn{2}{|l|}{ Race or ethnicity } \\
\hline White non-Hispanic & $352,603(24.1)$ \\
\hline Hispanic & $771,540(52.7)$ \\
\hline Black & $51,117(3.5)$ \\
\hline Asian & $206,251(14.1)$ \\
\hline Other $^{a}$ & $82,628(5.6)$ \\
\hline \multicolumn{2}{|l|}{ Maternal age at birth } \\
\hline$<18$ y & $34,005(2.3)$ \\
\hline 18 to $34 \mathrm{y}$ & $1,198,654$ (81.9) \\
\hline More than $34 \mathrm{y}$ & $231,456(15.8)$ \\
\hline \multicolumn{2}{|l|}{ Maternal education } \\
\hline$<12 \mathrm{y}$ & $403,563(27.6)$ \\
\hline $12 y$ & $334,503(22.9)$ \\
\hline More than $12 \mathrm{y}$ & $680,410(46.5)$ \\
\hline \multicolumn{2}{|l|}{ Payment for delivery } \\
\hline Medi-Cal $^{\mathrm{b}}$ & $673,017(46.0)$ \\
\hline Private insurance & $734,262(50.2)$ \\
\hline Self-pay & $13,580(0.9)$ \\
\hline Other $^{c}$ & $41,195(2.8)$ \\
\hline
\end{tabular}

Includes: American Indian, Hawaiian or Pacific Islander, other race, Two or more races and unknown race or ethnicity.

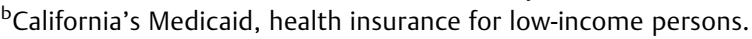
Includes: medically unattended birth, other government programs, Civilian Health and Medical Program of the Uniformed

Services/TRICARE, other payment and unknown.

\section{Results}

A total of 1,464,139 women met inclusion criteria (Fig. 1). The majority of the sample was white non-Hispanic $(24.1 \%)$ or Hispanic (50.2\%), between 18 and 34 years at delivery (81.9\%), had at least 12 years of education (69.4\%), and had private (50.2\%) or public insurance (46.0\%) for their delivery (- Table 1 ).

\section{Elevated AFP}

Women with elevated AFP were at increased risk of spontaneous and provider initiated PTB, with risk increasing for each degree of AFP elevation up to MoM 10.0 (-Table 2). While $1.1 \%$ of women with AFP $<2.5$ MoM delivered $<34$ weeks, $18.2 \%$ of women with AFP MoM between 6.0 and $<10.0$ had a birth $<34$ weeks (RR: $23.6,95 \% \mathrm{CI}$ : $15.8-$ 35.3). Women with elevated AFP were also at increased risk of obstetric complication, with AFP MoM 6.0 to $<10.0$ associated with the highest risk. Twenty-eight percent of women with AFP MoM 6.0 to $<10.0$ had both PTB and obstetric complication compared with $1.2 \%$ of women with AFP MoM $<2.5$ (RR: 27.4, 95\% CI: 17.6-41.4) (-Table 2). The risk of both PTB (< 37 weeks and $<34$ weeks) and obstetric complication among women with AFP MoM $\geq 10.0$ was increased above that of women without an elevated AFP, but less so relative to the other subgroups of elevated AFP (-Table 2 ).

\section{Elevated hCG}

Women with elevated hCG were at increased risk of both spontaneous and provider-initiated PTB $<37$ weeks and $<34$ weeks, with risks increasing with degree of hCG elevation (-Table 3). Of women with hCG MoM $\geq 10.0,50.8 \%$ had a PTB $<37$ weeks compared with $6.0 \%$ for hCG MoM $<2.5$ (RR: $16.2,95 \%$ CI: $9.8-26.8$ ) and a $23.0 \%$ rate of PTB $<34$ weeks compared with $1.1 \%$ for hCG MoM $<2.5$ (RR: 40.8, 95\% CI: 21.7-77.0). Increasing hCG MoM also conferred an increased risk of obstetric complication (-Table 3). For women with an hCG MoM $\geq 10.0,78.7 \%$ experienced either PTB or an obstetric complication compared with $15.5 \%$ with hCG MoM <2.5 (RR: 20.1, 95\% CI: 10.9-37.0) and 34.4\% experienced both a PTB and an obstetric complication compared with $1.2 \%$ with hCG MoM $<2.5$ (RR: $116.4,95 \% \mathrm{CI}$ : 58.3-232.4).

\section{Elevated uE3}

Women with elevated uE3 were at increased risk of both spontaneous and provider-initiated $\mathrm{PTB}<37$ and $<34$ weeks in the subgroup of women with uE3 MoM 2.5 to $<6.0$. Of these women, $14.8 \%$ had a PTB $<37$ weeks compared with $6.1 \%$ with uE3 MoM $<2.5$ (RR: 2.7, 95\% CI: 2.4-3.0) and 2.7\% had a PTB $<34$ weeks compared with $1.1 \%$ with $\mathrm{uE3} \mathrm{MoM}<2.5$ (RR: 2.7, 95\% CI: 2.1-3.4). Of the 1.4-million women in our sample, only 29 women had a $\mathrm{uE3}$ MoM between 6.0 and $<10.0$ and nine women had a uE3 MoM of 10.0 or more. Because of these small numbers, risk calculations were not performed for many of the outcomes ( - Table 4 ).

\section{Elevated INH}

Women with elevated INH were at increased risk of spontaneous and provider-initiated PTB $<37$ weeks and $<34$ weeks, with risk increasing with the degree of INH elevation up to MoM 10.0 (- Table 5). Women with elevated INH were also at increased risk of obstetric complication, which followed the same pattern as PTB. Women with an INH MoM 6.0 to $<10.0 \mathrm{MoM}$ and INH MoM $\geq 10.0$ had rates of any adverse outcome of $58.3 \%$ and $50.0 \%$, respectively, compared with $14.8 \%$ for INH MoM $<2.5$ (RR: 8.1, 95\% CI: 6.2-10.4 and RR: 5.8, 95\% CI: 3.8-8.8, respectively) (-Table 5).

\section{Two Elevated Analytes}

We identified 5,677 women with two elevated analytes $\geq 2.5$ MoM (-Table 6). The risk of PTB was increased for all combinations of elevated analytes (AFP and hCG, AFP and uE3, AFP and INH, hCG and uE3, hCG and INH, uE3 and INH) with the combinations of AFP and INH and hCG and INH conferring the greatest risk (RR: $10.5,95 \% \mathrm{CI}$ : 8.8-12.6 and RR: $10.8,95 \%$ CI: 5.6-20.8, respectively). The risk of obstetric complication was increased among women with the combinations of elevated AFP and hCG (38.9\% [RR: 5.3, 95\% CI: 4.76.0]), AFP and INH (45.9\% [RR: 7.1, 95\% CI: 6.0-8.5]), and hCG and INH (25\% (RR: 2.8, 95\% CI: 2.6-3.0)). Of women with elevated AFP and INH, 60.4\% experienced either a PTB or an obstetric complication (RR: 8.3, 95\% CI: 7.0-10.0) and 25.5\% experienced both a PTB and an obstetric complication (RR: 47.7, 95\% CI: 38.2-59.4). 
e372 Adverse Pregnancy Outcomes by Degree of Maternal Serum Analyte Elevation Yeaton-Massey et al.

Table 2 Risk of preterm birth or obstetric complication by AFP multiple of the median

\begin{tabular}{|c|c|c|c|c|}
\hline & \multicolumn{4}{|l|}{ AFP MoM } \\
\hline & $<2.5$ & 2.5 to $<6.0$ & 6.0 to $<10.0$ & 10.0 or more \\
\hline & $n(\%)$ & $n(\%)$ & $n(\%)$ & $n(\%)$ \\
\hline & RR $(95 \% \mathrm{Cl})$ & RR $(95 \% \mathrm{Cl})$ & RR $(95 \% \mathrm{Cl})$ & RR $(95 \% \mathrm{Cl})$ \\
\hline Sample & $1,411,294$ & 6,024 & 165 & 76 \\
\hline \multicolumn{5}{|l|}{ Gestation at birth } \\
\hline \multirow[t]{2}{*}{ Any $<37$ wk } & \multirow[t]{2}{*}{$84,772(6.0)$} & $1,253(20.8)$ & $55(33.3)$ & $14(18.4)$ \\
\hline & & $4.1(3.8-4.3)$ & $7.8(5.7-10.8)$ & $3.5(2.0-6.3)$ \\
\hline \multirow[t]{2}{*}{$<34$ wk } & \multirow[t]{2}{*}{$15,315(1.1)$} & $454(7.5)$ & $30(18.2)$ & $5(6.6)$ \\
\hline & & $8.0(7.3-8.8)$ & $23.6(15.835 .3)$ & $7.0(2.8-17.4)$ \\
\hline \multirow[t]{2}{*}{ Any $<37$ wk, PROM } & \multirow[t]{2}{*}{$15,716(1.1)$} & $274(4.6)$ & $14(8.5)$ & $3(4.0)$ \\
\hline & & $4.8(4.2-5.4)$ & $10.7(6.2-18.7)$ & $4.1(1.3-13.0)$ \\
\hline \multirow[t]{2}{*}{ Any $<37$ wk, spontaneous labor } & \multirow[t]{2}{*}{$36,814(2.6)$} & $626(10.4)$ & $27(16.4)$ & $9(11.8)$ \\
\hline & & $4.7(4.3-5.1)$ & $8.8(5.8-13.5)$ & $5.2(2.6-10.5)$ \\
\hline \multirow[t]{2}{*}{ Any $<37$ wk, provider initiated } & \multirow[t]{2}{*}{$18,841(1.3)$} & $232(3.9)$ & $11(6.7)$ & $1(1.3)$ \\
\hline & & $3.4(3.0-3.9)$ & $7.0(3.8-13.1)$ & a \\
\hline \multirow[t]{2}{*}{37 wk or more } & $1,326,522(94.0)$ & 4,771 (79.2) & $110(66.7)$ & $62(81.6)$ \\
\hline & Reference & & & \\
\hline \multicolumn{5}{|l|}{ Obstetric complication } \\
\hline \multirow[t]{2}{*}{ None } & $1,259,222(89.2)$ & $4,441(73.7)$ & $99(60.0)$ & $60(79.0)$ \\
\hline & Reference & & & \\
\hline \multirow[t]{2}{*}{ Any } & \multirow[t]{2}{*}{$152,072(10.8)$} & $1,583(26.3)$ & $66(40.0)$ & $16(21.1)$ \\
\hline & & $2.9(2.8-3.1)$ & $5.5(4.0-7.5)$ & $2.2(1.3-3.8)$ \\
\hline \multirow[t]{2}{*}{ Preeclampsia } & \multirow[t]{2}{*}{$38,268(2.7)$} & $437(7.3)$ & $19(11.5)$ & $4(5.3)$ \\
\hline & & $3.2(2.9-3.5)$ & $6.3(3.9-10.3)$ & $2.1(0.8-6.0)$ \\
\hline \multirow[t]{2}{*}{ Placental abruption } & \multirow[t]{2}{*}{$9,892(0.7)$} & $123(2.0)$ & $8(4.9)$ & $0(0.0)$ \\
\hline & & $3.5(2.9-4.2)$ & $10.3(5.0-21.1)$ & $\mathrm{a}$ \\
\hline \multirow[t]{2}{*}{ Small for gestational age } & \multirow[t]{2}{*}{$112,941(8.0)$} & $1,235(20.5)$ & $53(32.1)$ & $13(17.1)$ \\
\hline & & $3.1(2.9-3.3)$ & $6.0(4.3-8.3)$ & $2.4(1.3-4.4)$ \\
\hline \multirow[t]{2}{*}{ Any adverse outcome } & \multirow[t]{2}{*}{$219,942(15.6)$} & $2,290(38.0)$ & $93(56.4)$ & $26(34.2)$ \\
\hline & & $3.3(3.1-3.5)$ & $7.0(5.1-9.5)$ & $2.8(1.8-4.5)$ \\
\hline \multirow[t]{2}{*}{ Preterm birth and obstetric complication } & \multirow[t]{2}{*}{$16,902(1.2)$} & $546(9.1)$ & $28(17.0)$ & $4(5.3)$ \\
\hline & & $10.0(9.2-10.9)$ & $27.4(17.7-42.3)$ & $5.6(2.0-15.6)$ \\
\hline \multirow[t]{2}{*}{ Neither preterm birth nor obstetric complication } & $1,191,352(84.4)$ & $3,734(62.0)$ & $72(43.6)$ & $50(65.8)$ \\
\hline & Reference & & & \\
\hline
\end{tabular}

Abbreviations: AFP, $\alpha$-fetoprotein; $\mathrm{Cl}$, confidence interval; MoM, multiple of the median; PROM, premature rupture of the membranes; RR, relative risk. ${ }^{a}$ Not calculated when $n<$ three.

\section{Discussion}

In this large, retrospective study of pregnancy outcomes based on elevated maternal serum analytes without major structural anomalies or aneuploidy, we examined rates of PTB and obstetric complication by degree of second-trimester maternal serum analyte elevation. Our analysis demonstrated that most risks of adverse pregnancy outcome increase as the maternal serum analyte MoM increases, and that elevations in hCG and INH appear to be associated with underlying pathology that confers pregnancy risks that are far greater than elevations of AFP.

While previous studies have characterized outcomes for pregnancies complicated by abnormal maternal serum analytes, these studies do not provide data stratified by degree of analyte elevation (i.e., MoM subgroup > 2.5). ${ }^{1,4-6,10-13,15}$ By using a large dataset, we were able to assess for uncommon analyte elevations with sufficient sample size to analyze based on analyte subgroups. Our analysis provides specific information based on analyte and elevation subgroup to 
Table 3 Risk of preterm birth or obstetric complication by hCG multiple of the median

\begin{tabular}{|c|c|c|c|c|}
\hline & \multicolumn{4}{|l|}{ hCG MoM } \\
\hline & $<2.5$ & 2.5 to $<6.0$ & 6.0 to $<10.0$ & 10.0 or more \\
\hline & $n(\%)$ & $n(\%)$ & $n(\%)$ & $n(\%)$ \\
\hline & RR $(95 \% \mathrm{CI})$ & RR (95\% Cl) & $\mathrm{RR}(95 \% \mathrm{Cl})$ & $\mathrm{RR}(95 \% \mathrm{CI})$ \\
\hline Sample & $1,372,653$ & 44,359 & 486 & 61 \\
\hline \multicolumn{5}{|l|}{ Gestation at birth } \\
\hline \multirow[t]{2}{*}{ Any $<37$ wk } & \multirow[t]{2}{*}{$82,182(6.0)$} & $3,782(8.5)$ & $99(20.4)$ & $31(50.8)$ \\
\hline & & $1.4(1.4-1.5)$ & $4.0(3.2-5.0)$ & $16.2(9.8-26.8)$ \\
\hline \multirow[t]{2}{*}{$<34 \mathrm{wk}$} & \multirow[t]{2}{*}{$14,739(1.1)$} & $1,009(2.3)$ & $42(8.6)$ & $14(23.0)$ \\
\hline & & $2.1(2.0-2.2)$ & $9.5(6.9-13.0)$ & $40.8(21.7-77.0)$ \\
\hline \multirow[t]{2}{*}{ Any $<37$ wk, PROM } & \multirow[t]{2}{*}{$15,340(1.1)$} & $649(1.5)$ & $14(2.9)$ & $4(6.6)$ \\
\hline & & $1.3(1.2-1.4)$ & $3.0(1.8-5.2)$ & $11.2(4.0-31.8)$ \\
\hline \multirow[t]{2}{*}{ Any $<37$ wk, spontaneous labor } & \multirow[t]{2}{*}{$35,577(2.6)$} & $1,829(4.1)$ & $47(9.7)$ & $23(37.7)$ \\
\hline & & $1.6(1.5-1.7)$ & $4.4(3.3-6.0)$ & $27.8(16.1-47.8)$ \\
\hline \multirow[t]{2}{*}{ Any $<37$ wk, provider initiated } & \multirow[t]{2}{*}{$18,176(1.3)$} & $879(2.0)$ & $27(5.6)$ & $3(4.9)$ \\
\hline & & $1.5(1.4-1.6)$ & $4.9(3.4-7.3)$ & $7.1(2.2-23.3)$ \\
\hline \multirow[t]{2}{*}{37 wk or more } & $1,290,471(94.0)$ & $40,577(91.5)$ & $387(79.6)$ & $30(49.2)$ \\
\hline & Reference & & & \\
\hline \multicolumn{5}{|l|}{ Obstetric complication } \\
\hline \multirow[t]{2}{*}{ None } & $1,225,506(89.3)$ & $37,962(85.6)$ & $331(68.1)$ & $23(37.7)$ \\
\hline & Reference & & & \\
\hline \multirow[t]{2}{*}{ Any } & \multirow[t]{2}{*}{$147,147(10.7)$} & $6,397(14.4)$ & $155(31.9)$ & $38(62.3)$ \\
\hline & & $1.4(1.4-1.4)$ & $3.9(3.2-4.7)$ & $13.8(8.2-23.1)$ \\
\hline \multirow[t]{2}{*}{ Preeclampsia } & \multirow[t]{2}{*}{$36,235(2.6)$} & $2,397(5.4)$ & $73(15.0)$ & $23(37.7)$ \\
\hline & & $2.1(2.0-2.1)$ & $7.4(5.8-9.6)$ & $33.8(19.0-60.2)$ \\
\hline \multirow[t]{2}{*}{ Placental abruption } & \multirow[t]{2}{*}{$9,562(0.7)$} & $440(1.0)$ & $13(2.7)$ & $8(13.1)$ \\
\hline & & $1.5(1.3-1.6)$ & $5.0(2.9-8.7)$ & 44.5 (19.9-99.6) \\
\hline \multirow[t]{2}{*}{ Small for gestational age } & \multirow[t]{2}{*}{$109,888(8.0)$} & $4,227(9.5)$ & $101(20.8)$ & $26(42.6)$ \\
\hline & & $1.2(1.2-1.3)$ & $3.4(2.7-4.2)$ & $12.6(7.2-22.1)$ \\
\hline \multirow[t]{2}{*}{ Any adverse outcome } & \multirow[t]{2}{*}{$213,262(15.5)$} & $8,849(20.0)$ & $192(39.5)$ & $48(78.7)$ \\
\hline & & $1.3(1.3-1.4)$ & $3.5(3.0-4.3)$ & $20.1(10.9-37.0)$ \\
\hline \multirow{2}{*}{$\begin{array}{r}\text { Preterm birth and } \\
\text { obstetric complication }\end{array}$} & \multirow[t]{2}{*}{$16,067(1.2)$} & $1,330(3.0)$ & $62(12.8)$ & $21(34.4)$ \\
\hline & & $2.6(2.4-2.7)$ & $15.2(11.5-19.9)$ & $116.4(58.3-232.4)$ \\
\hline \multirow[t]{2}{*}{$\begin{array}{r}\text { Neither preterm birth nor } \\
\text { obstetric complication }\end{array}$} & $1,159,391(84.5)$ & $35,510(80.1)$ & $294(60.5)$ & $13(21.3)$ \\
\hline & Reference & & & \\
\hline
\end{tabular}

Abbreviations: $\mathrm{Cl}$, confidence interval; hCG, human chorionic gonadotropin; MoM, multiple of the median; PROM, premature rupture of the membranes; RR, relative risk.

${ }^{a}$ Not calculated when $n<$ three.

better allow for personalized patient counseling and guide antepartum surveillance for a unique population at high risk of adverse pregnancy outcome.

Our findings are consistent with prior studies demonstrating adverse pregnancy outcomes with elevated hCG; we identified an ongoing increase in risk based on degree of MoM elevation. ${ }^{10,11,17,25}$ The cause of isolated elevation of hCG is not entirely understood; however, it is known that hypoxic cytotrophoblasts proliferate at a higher rate thus producing higher degrees of serum hCG. ${ }^{25}$ It is possible that cytotrophoblast hypoxia impairs implantation, which in turn leads to poor placentation and related adverse pregnancy outcomes. Studies of placental pathology demonstrate an association between chorionic villitis and vascular lesions of the placenta that allow for increased AFP diffusion from fetal to maternal circulation (i.e., following a concentration 
Table 4 Risk of preterm birth or obstetric complication by uE3 multiple of the median

\begin{tabular}{|c|c|c|c|c|}
\hline & \multicolumn{4}{|l|}{ uE3 MoM } \\
\hline & $<2.5$ & 2.5 to $<6.0$ & 6.0 to $<10.0$ & 10.0 or more \\
\hline & $n(\%)$ & $n(\%)$ & $n(\%)$ & $n(\%)$ \\
\hline & RR (95\% CI) & RR (95\% Cl) & RR (95\% Cl) & RR (95\% CI) \\
\hline Sample & $1,413,598$ & 2,435 & 29 & 9 \\
\hline \multicolumn{5}{|l|}{ Gestation at birth } \\
\hline \multirow[t]{2}{*}{ Any $<37$ wk } & \multirow[t]{2}{*}{$85,639(6.1)$} & $361(14.8)$ & $2(6.9)$ & $1(11.1)$ \\
\hline & & $2.7(2.4-3.0)$ & $\mathrm{a}$ & $\mathrm{a}$ \\
\hline \multirow[t]{2}{*}{$<34$ wk } & \multirow[t]{2}{*}{$15,722(1.1)$} & $66(2.7)$ & $0(0.0)$ & $0(0.0)$ \\
\hline & & $2.7(2.1-3.4)$ & $\mathrm{a}$ & a \\
\hline \multirow[t]{2}{*}{ Any < 37 wk, PROM } & \multirow[t]{2}{*}{$15,961(1.1)$} & $33(1.4)$ & $0(0.0)$ & $0(0.0)$ \\
\hline & & $1.3(0.9-1.9)$ & $\mathrm{a}$ & $\mathrm{a}$ \\
\hline \multirow[t]{2}{*}{ Any $<37$ wk, spontaneous labor } & \multirow[t]{2}{*}{$37,344(2.6)$} & $96(3.9)$ & $1(3.5)$ & $1(11.1)$ \\
\hline & & $1.6(1.3-2.0)$ & $\mathrm{a}$ & $\mathrm{a}$ \\
\hline \multirow[t]{2}{*}{ Any $<37$ wk, provider initiated } & \multirow[t]{2}{*}{$18,948(1.3)$} & $111(4.6)$ & $1(3.5)$ & $0(0.0)$ \\
\hline & & $3.7(3.1-4.5)$ & $\mathrm{a}$ & $\mathrm{a}$ \\
\hline \multirow[t]{2}{*}{37 wk or more } & $1,327,959$ (93.9) & $2,074(85.2)$ & $27(93.1)$ & $8(88.9)$ \\
\hline & Reference & & & \\
\hline \multicolumn{5}{|l|}{ Obstetric complication } \\
\hline \multirow[t]{2}{*}{ None } & $1,260,263(89.2)$ & $2,203(90.5)$ & $27(93.1)$ & $7(77.8)$ \\
\hline & Reference & & & \\
\hline \multirow[t]{2}{*}{ Any } & \multirow[t]{2}{*}{$153,335(10.9)$} & $232(9.5)$ & $2(6.9)$ & $2(22.2)$ \\
\hline & & $0.9(0.8-1.0)^{\mathrm{b}}$ & $\mathrm{a}$ & $\mathrm{a}$ \\
\hline \multirow[t]{2}{*}{ Preeclampsia } & \multirow[t]{2}{*}{$38,618(2.7)$} & $73(3.0)$ & $0(0.0)$ & $0(0.0)$ \\
\hline & & $1.1(0.9-1.4)$ & $\mathrm{a}$ & $\mathrm{a}$ \\
\hline \multirow[t]{2}{*}{ Placental abruption } & \multirow[t]{2}{*}{$9,994(0.7)$} & $16(0.7)$ & $0(0.0)$ & $0(0.0)$ \\
\hline & & $0.9(0.6-1.5)$ & $\mathrm{a}$ & $\mathrm{a}$ \\
\hline \multirow[t]{2}{*}{ Small for gestational age } & \multirow[t]{2}{*}{$113,952(8.1)$} & $157(6.5)$ & $2(6.9)$ & $2(22.2)$ \\
\hline & & $0.8(0.7-0.9)$ & a & a \\
\hline \multirow[t]{2}{*}{ Any adverse outcome } & \multirow[t]{2}{*}{$221,545(15.7)$} & 558 (22.9) & $3(10.3)$ & $3(33.3)$ \\
\hline & & $1.6(1.5-1.8)$ & $0.6(0.2-2.1)$ & $2.6(0.7-10.8)$ \\
\hline \multirow[t]{2}{*}{ Preterm birth and obstetric complication } & \multirow[t]{2}{*}{$17,429(1.2)$} & $35(1.4)$ & $1(3.5)$ & $0(0.0)$ \\
\hline & & $1.3(0.9-1.8)$ & $\mathrm{a}$ & $\mathrm{a}$ \\
\hline \multirow[t]{2}{*}{ Neither preterm birth nor obstetric complication } & $1,192,053(84.3)$ & $1,877(77.1)$ & $26(89.7)$ & $6(66.7)$ \\
\hline & Reference & & & \\
\hline
\end{tabular}

Abbreviations: $\mathrm{Cl}$, confidence interval; MoM, multiple of the median; PROM, premature rupture of the membranes; RR, relative risk; uE3, unconjugated estriol.

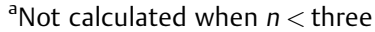

${ }^{\mathrm{b}} p$-value $<0.05$.

gradient).${ }^{14}$ In the setting of isolated elevation of AFP, there is less risk increase for PTB or obstetric complication for $\mathrm{MoM} \geq 10.0$ compared with MoM 6.0 to $<10.0$. One possible explanation for this is that our data only capture live births thus missing any pregnancies with elevated AFP that ended in termination, miscarriage, or stillbirth. It is possible that pregnancies with an AFP MoM $\geq 10.0$ have a lower rate of PTB and obstetric complication because they had a lower live birth rate. Our cohort excluded cases with major structural birth defects, a category that included Finnish nephrosis, eliminating this known source of elevated AFP.

In this large cohort of women undergoing prenatal screening with maternal serum analytes, we found a marked increase in risk of PTB and obstetric complication for women with elevated second-trimester maternal serum analytes that peaked at MoM 6.0 to $<10.0$ MoM for AFP and inhibin 
Table 5 Risk of preterm birth or obstetric complication by INH multiple of the median

\begin{tabular}{|c|c|c|c|c|}
\hline & \multicolumn{4}{|l|}{ INH MoM } \\
\hline & $<2.5$ & 2.5 to $<6.0$ & 6.0 to $<10.0$ & 10.0 or more \\
\hline & $n(\%)$ & $n(\%)$ & $n(\%)$ & $n(\%)$ \\
\hline & RR (95\% Cl) & RR (95\% CI) & RR (95\% CI) & RR (95\% CI) \\
\hline Sample & 728,944 & 10,221 & 240 & 86 \\
\hline \multicolumn{5}{|l|}{ Gestation at birth } \\
\hline \multirow[t]{2}{*}{ Any $<37$ wk } & \multirow[t]{2}{*}{$38,679(5.3)$} & $1,349(13.2)$ & $90(37.5)$ & $25(29.1)$ \\
\hline & & $2.7(2.5-2.8)$ & $10.7(8.2-13.9)$ & $7.3(4.6-11.6)$ \\
\hline \multirow[t]{2}{*}{$<34$ wk } & \multirow[t]{2}{*}{$7,535(1.0)$} & 504 (4.9) & $39(16.3)$ & $15(17.4)$ \\
\hline & & $4.9(4.5-5.4)$ & $23.7(16.7-33.7)$ & $22.5(12.8-39.5)$ \\
\hline \multirow[t]{2}{*}{ Any $<37$ wk, PROM } & \multirow[t]{2}{*}{$8,669(1.2)$} & $232(2.3)$ & $18(7.5)$ & $3(3.5)$ \\
\hline & & $2.1(1.8-2.3)$ & $9.5(5.9-15.5)$ & $3.9(1.2-12.5)$ \\
\hline \multirow[t]{2}{*}{ Any $<37$ wk, spontaneous labor } & \multirow[t]{2}{*}{$19,813(2.7)$} & $768(7.5)$ & $46(19.2)$ & $15(17.4)$ \\
\hline & & $2.9(2.7-3.2)$ & $10.7(7.7-14.8)$ & $8.6(4.9-15.1)$ \\
\hline \multirow[t]{2}{*}{ Any $<37$ wk, provider initiated } & \multirow[t]{2}{*}{$6,312(0.9)$} & $280(2.7)$ & $23(9.6)$ & $6(7.0)$ \\
\hline & & $3.3(3.0-3.8)$ & $16.7(10.8-25.9)$ & $10.7(4.6-24.9)$ \\
\hline \multirow[t]{2}{*}{37 wk or more } & $690,265(94.7)$ & $8,872(86.8)$ & $150(62.5)$ & $61(70.9)$ \\
\hline & Reference & & & \\
\hline \multicolumn{5}{|l|}{ Obstetric complication } \\
\hline \multirow[t]{2}{*}{ None } & $651,542(89.4)$ & 7,939 (77.7) & $126(52.5)$ & $50(58.1)$ \\
\hline & Reference & & & \\
\hline \multirow[t]{2}{*}{ ny } & \multirow[t]{2}{*}{$77,402(10.6)$} & $2,282(22.3)$ & $114(47.5)$ & 36 (41.9) \\
\hline & & $2.4(2.3-2.5)$ & $7.6(5.9-9.8)$ & $6.1(3.9-9.3)$ \\
\hline \multirow[t]{2}{*}{ Preeclampsia } & \multirow[t]{2}{*}{$19,605(2.7)$} & $1,030(10.1)$ & $58(24.2)$ & $20(23.3)$ \\
\hline & & $4.1(3.9-4.4)$ & $15.3(11.2-20.8)$ & $13.3(7.9-22.3)$ \\
\hline \multirow[t]{2}{*}{ Placental abruption } & \multirow[t]{2}{*}{$5,159(0.7)$} & $163(1.6)$ & $11(4.6)$ & $3(3.5)$ \\
\hline & & $2.5(2.2-3.0)$ & $11.0(5.9-20.4)$ & $7.6(2.4-24.3)$ \\
\hline \multirow[t]{2}{*}{ Small for gestational age } & \multirow[t]{2}{*}{$56,944(7.8)$} & $1,468(14.4)$ & $76(31.7)$ & $25(29.1)$ \\
\hline & & $2.1(2.0-2.2)$ & $6.9(5.2-9.2)$ & $5.7(3.5-9.2)$ \\
\hline \multirow[t]{2}{*}{ Any adverse outcome } & \multirow[t]{2}{*}{$107,642(14.8)$} & $2,920(28.6)$ & $140(58.3)$ & $43(50.0)$ \\
\hline & & $2.3(2.2-2.4)$ & $8.1(6.2-10.4)$ & $5.8(3.8-8.8)$ \\
\hline \multirow[t]{2}{*}{ Preterm birth and obstetric complication } & \multirow[t]{2}{*}{$8,429(1.2)$} & $711(7.0)$ & $64(26.7)$ & $18(20.9)$ \\
\hline & & $6.7(6.2-7.2)$ & $46.8(34.2-64.0)$ & $30.8(17.7-53.3)$ \\
\hline \multirow[t]{2}{*}{ Neither preterm birth nor obstetric complication } & $621,302(85.2)$ & $7,301(71.4)$ & $100(41.7)$ & $43(50.0)$ \\
\hline & Reference & & & \\
\hline
\end{tabular}

Abbreviations: $\mathrm{Cl}$, confidence interval; INH, inhibin A; MoM, multiple of the median; PROM, premature rupture of the membranes; RR, relative risk.

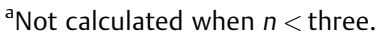

and continued to rise for hCG MoM $\geq 10.0$. Our data confirm the known association between elevated maternal serum analytes and adverse obstetric outcome and add additional information regarding the association between degree of analyte elevation and risk of PTB and obstetric complication.

Our study is not without limitations. While we utilized the Genetic Disease Screening Program California Chromosomal Defect Registry to exclude cases with known aneuploidy, we cannot ensure that all patients included in our cohort had euploid pregnancies (e.g., had prenatal or post- natal diagnostic testing to confirm a euploid pregnancy). Given this limitation, it is possible that some small portion of pregnancies in our sample was in fact aneuploid. If this is the case, we would anticipate that our data might slightly overestimate risk given the association between aneuploidy and adverse pregnancy outcomes. We acknowledge that since this is a large dataset, there is risk of error due to misclassification of outcomes. It is also possible that not all adverse obstetric outcomes were captured. However, if this is the case, we would expect misclassification of adverse 


\begin{tabular}{|c|c|c|c|c|c|c|c|c|c|c|c|c|c|c|c|c|c|c|c|c|c|c|c|c|c|c|c|}
\hline 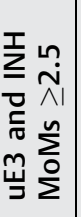 & $\stackrel{Ð}{\varrho}$ & 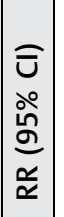 & $\hat{m}$ & 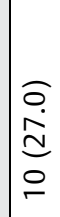 & 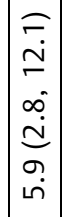 & $\frac{a}{\infty}$ & $\begin{array}{l}\tilde{\sigma} \\
\tilde{n} \\
\dot{0} \\
\ddot{m} \\
0 \\
\dot{0} \\
\dot{0}\end{array}$ & $\begin{array}{c}\underset{r}{d} \\
\stackrel{c}{d} \\
r\end{array}$ & ro & 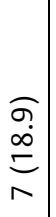 & 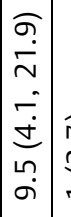 & $\begin{array}{c}\underset{\mathfrak{d}}{\mathfrak{d}} \\
-\end{array}$ & ? & 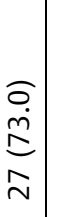 & & $\begin{array}{l}\widehat{\hat{n}} \\
\dot{\infty} \\
\infty \\
m \\
m\end{array}$ & & 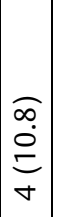 & 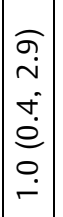 & $\begin{array}{l}\widehat{\partial} \\
\dot{\theta} \\
0\end{array}$ & 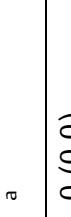 & 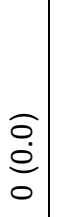 & 0 & $\begin{array}{l} \\
\widehat{\infty} \\
\dot{0} \\
\stackrel{\sigma}{\sigma} \\
\sigma\end{array}$ & 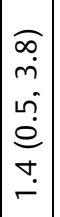 & 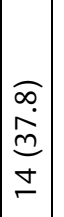 & $\begin{array}{l}\widehat{n} \\
6 \\
\\
\\
m \\
m \\
m\end{array}$ \\
\hline 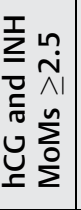 & $\begin{array}{l}\sqrt{0} \\
=\end{array}$ & 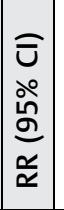 & \begin{tabular}{|l} 
\\
\\
\multirow{2}{*}{} \\
$\infty$ \\
$\infty$ \\
$m^{\prime}$
\end{tabular} & 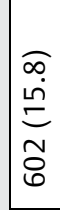 & 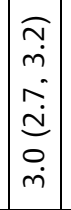 & 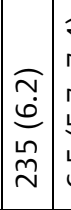 & 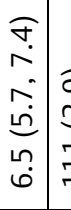 & $\begin{array}{l}a \\
\underset{a}{d} \\
\underline{\Xi} \\
\Xi\end{array}$ & 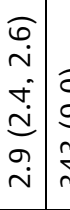 & $\begin{array}{l} \\
\stackrel{o}{o} \\
\dot{g} \\
\stackrel{m}{m}\end{array}$ & 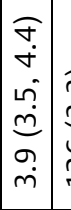 & 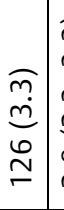 & $\begin{array}{c}\tilde{m} \\
m \\
m \\
\stackrel{d}{d} \\
\infty \\
\dot{\sim}\end{array}$ & 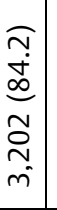 & & 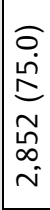 & & 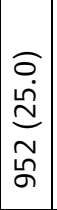 & 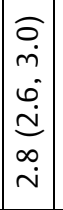 & 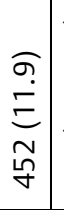 & 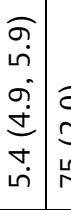 & $\begin{array}{l}\underset{\partial}{0} \\
\stackrel{d}{d} \\
\stackrel{n}{n}\end{array}$ & 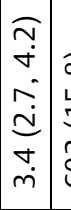 & 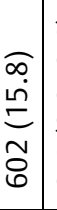 & 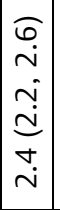 & 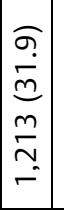 & 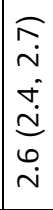 \\
\hline 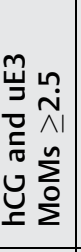 & 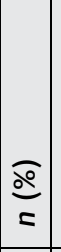 & $\begin{array}{l}\bar{\cup} \\
\stackrel{\circ}{2} \\
\stackrel{\alpha}{\alpha} \\
\frac{\alpha}{\alpha}\end{array}$ & m & 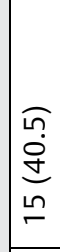 & 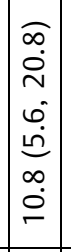 & $\begin{array}{c}\sigma \\
\infty \\
\infty \\
\curvearrowright \\
\sim\end{array}$ & 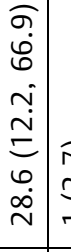 & $\begin{array}{c}\underset{a}{c} \\
\stackrel{d}{d} \\
-\end{array}$ & 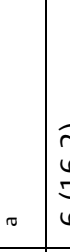 & 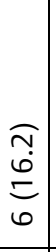 & 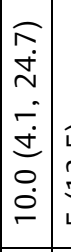 & 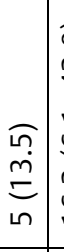 & 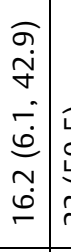 & 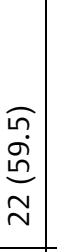 & & $\begin{array}{l}\widehat{\infty} \\
\stackrel{\infty}{\infty} \\
\infty \\
\bar{m} \\
\bar{m}\end{array}$ & & 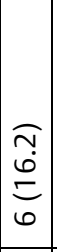 & 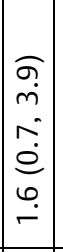 & $\begin{array}{l}\underset{\sigma}{\vec{C}} \\
\stackrel{\sim}{N}\end{array}$ & ת & $\begin{array}{l}\underset{\mathfrak{a}}{\mathfrak{d}} \\
-\end{array}$ & م & $\begin{array}{l} \\
\widehat{\infty} \\
\dot{0} \\
\stackrel{\sigma}{\sigma} \\
\vec{\sigma}\end{array}$ & 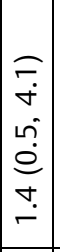 & 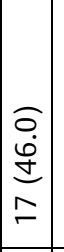 & 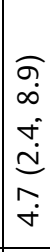 \\
\hline 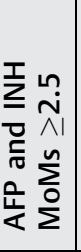 & 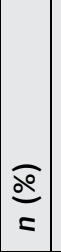 & $\begin{array}{l}\bar{\sigma} \\
\stackrel{\circ}{0} \\
\hat{\sigma} \\
\frac{\alpha}{\alpha}\end{array}$ & 농 & 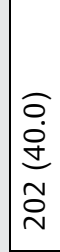 & 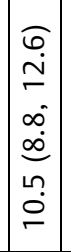 & 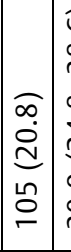 & 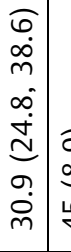 & \begin{tabular}{c|} 
\\
$\sigma$ \\
$\infty$ \\
$\infty$ \\
$\dot{q}$
\end{tabular} & 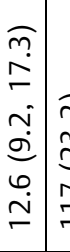 & 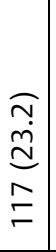 & 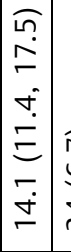 & $\begin{array}{c} \\
\widetilde{\sigma} \\
\dot{\theta} \\
\dot{b} \\
\dot{m}\end{array}$ & 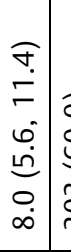 & $\begin{array}{l}o \\
\dot{0} \\
\dot{\theta} \\
m \\
\dot{m}\end{array}$ & & $\begin{array}{l}\widetilde{c} \\
\dot{\dot{b}} \\
\dot{n} \\
\stackrel{n}{N}\end{array}$ & & 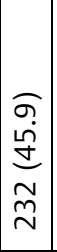 & 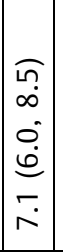 & 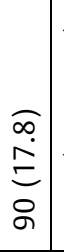 & 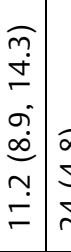 & $\begin{array}{l} \\
\widehat{\infty} \\
\dot{ \pm} \\
\stackrel{\Delta}{\sim}\end{array}$ & 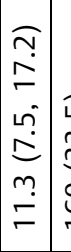 & 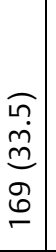 & 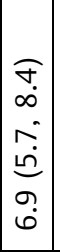 & 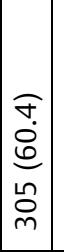 & 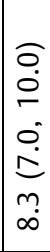 \\
\hline 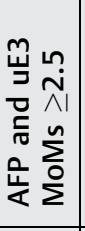 & 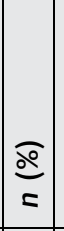 & 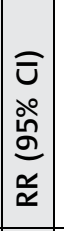 & ${ }_{\infty}$ & 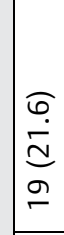 & 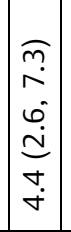 & $\frac{0}{0}$ & 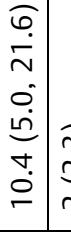 & $\begin{array}{l}\widetilde{m} \\
\underset{\sim}{\sim} \\
\sim\end{array}$ & ro & $\begin{array}{l}\overparen{\mathfrak{n}} \\
\stackrel{\Xi}{\Xi} \\
=\end{array}$ & 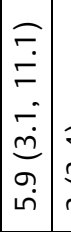 & $\begin{array}{c}\widehat{\sigma} \\
\stackrel{m}{m} \\
m\end{array}$ & $\begin{array}{c}\hat{\sigma} \\
\sigma \\
0 \\
= \\
\bar{\Xi} \\
\bar{m}\end{array}$ & 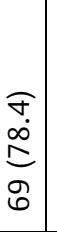 & & 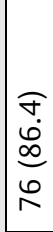 & & 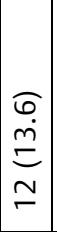 & 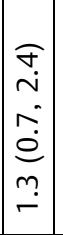 & 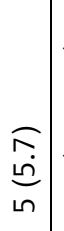 & 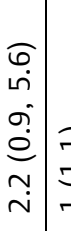 & בְ. & . & $\begin{array}{l}\widehat{o} \\
\stackrel{0}{\varphi} \\
0\end{array}$ & 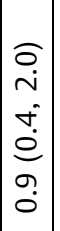 & 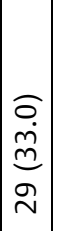 & 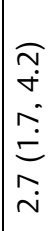 \\
\hline 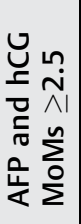 & $\stackrel{\widehat{Ð}}{=}$ & 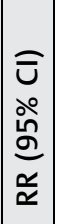 & 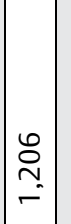 & 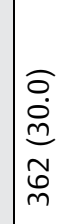 & $\mid \begin{array}{c}\tilde{T} \\
\hat{i} \\
0 \\
0 \\
\dot{\theta} \\
0 \\
\dot{\varphi} \\
0\end{array}$ & 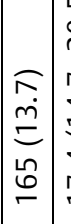 & 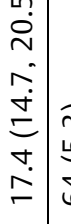 & \begin{tabular}{c|c} 
& \\
$\widetilde{m}$ & \\
$\stackrel{n}{n}$ & \\
$\tilde{\sigma}$ &
\end{tabular} & $\begin{array}{c}\widehat{m} \\
\infty \\
0 \\
0 \\
\text { n் } \\
\vdots \\
\dot{b}\end{array}$ & 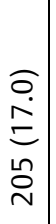 & $\mid \begin{array}{c}\tilde{m} \\
0 \\
0 \\
0 \\
\tilde{D} \\
0 \\
\infty \\
\infty \\
0\end{array}$ & 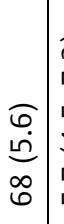 & 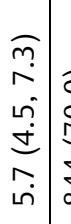 & 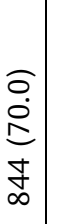 & & $\begin{array}{l}\widehat{\sigma} \\
\bar{\sigma} \\
\hat{n}\end{array}$ & & 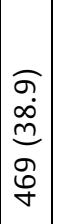 & 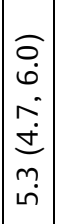 & 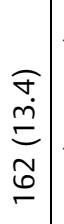 & 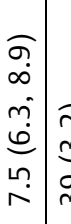 & $\begin{array}{l}\text { गิ } \\
\stackrel{m}{\sigma} \\
\stackrel{m}{m}\end{array}$ & 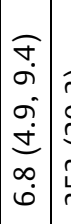 & 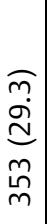 & 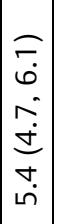 & 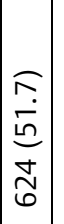 & 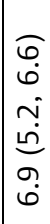 \\
\hline 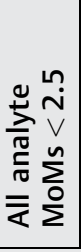 & $\stackrel{\widehat{\varrho}}{=}$ & 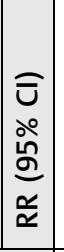 & 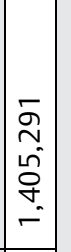 & 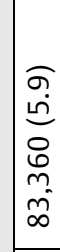 & & 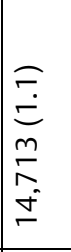 & & 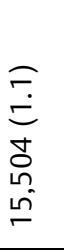 & & 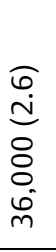 & & 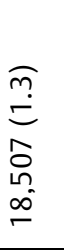 & & 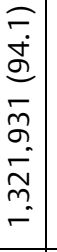 & 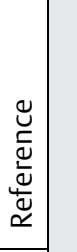 & 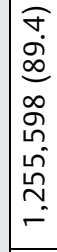 & 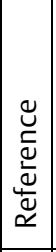 & 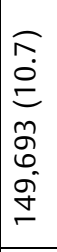 & & $\begin{array}{l}\widehat{0} \\
\stackrel{i}{d} \\
0 \\
\underline{N} \\
\hat{b} \\
m\end{array}$ & & $\begin{array}{l}\hat{\widehat{T}} \\
\dot{0} \\
\dot{b} \\
\stackrel{N}{N} \\
\tilde{\sigma}^{\circ}\end{array}$ & & 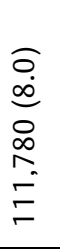 & & 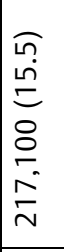 & \\
\hline & & & $\begin{array}{l}\frac{0}{a} \\
\frac{\vec{E}}{\sim} \\
\stackrel{\sim}{n}\end{array}$ & 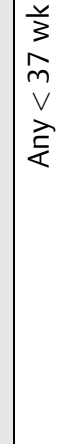 & & 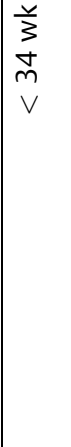 & & 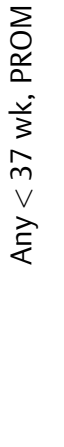 & & 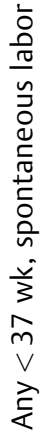 & & 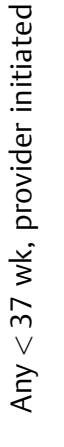 & & 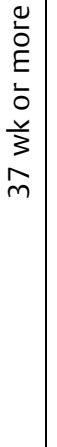 & & $\begin{array}{l}0 \\
\tilde{o} \\
z\end{array}$ & & 安 & & 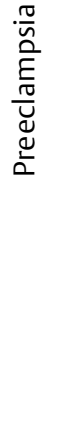 & & 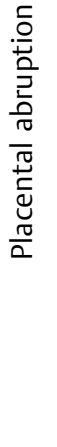 & & 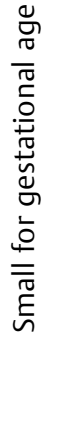 & & 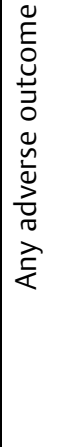 & \\
\hline
\end{tabular}




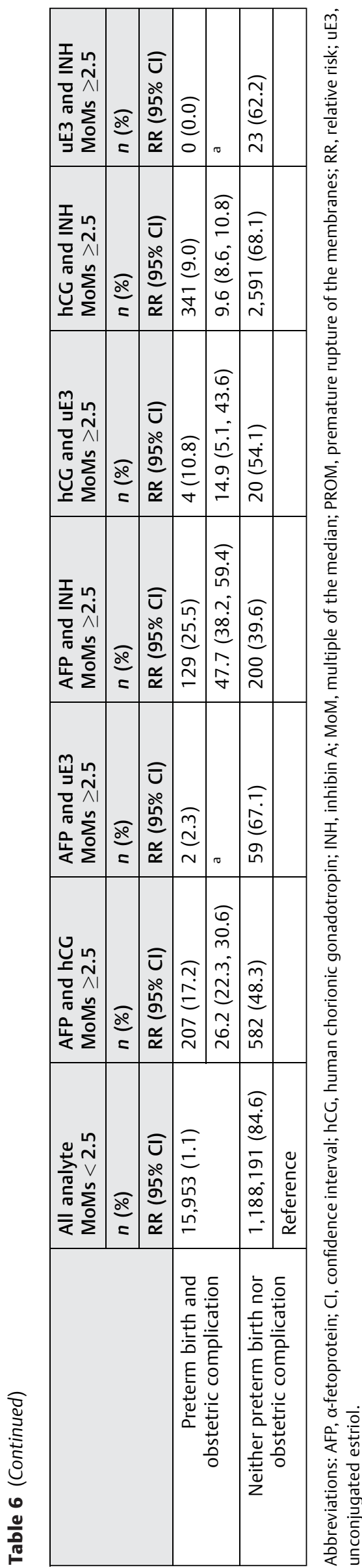

outcomes to be evenly distributed for women in the elevated analyte subgroups. Pregnancies that ended in stillbirth were not included in available data, so we are missing the outcome of stillbirth in our assessment of obstetric complication. Lastly, we were unable to identify women with more than one live birth in the cohort and thus were unable to adjust for this. In spite of these limitations, our data provide new information on RR of PTB and obstetric complication stratified by degree of maternal serum analyte elevation and fill a gap in the literature.

In euploid, structurally normal fetuses, all analyte elevations $>2.5$ MoM confer an increased risk of PTB and, except for $\mathrm{uE3}$, obstetric complication. Maternal serum analyte elevations may serve as a marker of underlying placental pathology and confer risk of adverse pregnancy outcome. Risks of PTB and obstetric complication are not uniformly linear, thus these detailed data for individual analytes by subgroup can help guide patient counseling and antenatal management.

\section{Author Contributions}

The authors certify that they have made substantial contributions to the conception and design (AYM, RJB, LR, LJP, DJL), or analysis (RJB, LJP) and interpretation of data (AYM, RJB, LR, LJP, DJL). In addition, they certify their contributions to drafting the article or revising it critically for important intellectual content (AYM, RJB, LR, LJP, DJL). The authors give final approval to the version to be published and agree to be accountable for all aspects of the work in ensuring that questions related to the accuracy or integrity of any part of the work are appropriately investigated and resolved (AYM, RJB, LR, LJP, DJL).

\section{Funding Information}

This work was supported in part by the University of California, San Francisco California Preterm Birth Initiative. Data from the California Prenatal and Newborn Screening Programs were obtained through the California Biobank Program (Screening Information System request no. 476). Data were obtained with an agreement that the California Department of Public Health is not responsible for the results or conclusions drawn by the authors of this publication.

\section{Conflict of Interest}

None.

Acknowledgments

This work was supported in part by the University of California, San Francisco California Preterm Birth Initiative.

\section{References}

1 Heinonen S, Ryynänen M, Kirkinen P, Saarikoski S. Elevated midtrimester maternal serum hCG in chromosomally normal pregnancies is associated with preeclampsia and velamentous umbilical cord insertion. Am J Perinatol 1996;13(07):437-441

2 Taché V, Baer RJ, Currier RJ, et al. . Population-based biomarker screening and the development of severe preeclampsia in California. Am J Obstet Gynecol 2014;211(04):377.e1-377.e8 
3 Waller DK, Lustig LS, Cunningham GC, Feuchtbaum LB, Hook EB. The association between maternal serum alpha-fetoprotein and preterm birth, small for gestational age infants, preeclampsia, and placental complications. Obstet Gynecol 1996;88(05): 816-822

4 Jelliffe-Pawlowski LL, Shaw GM, Currier RJ, et al. . Association of early-preterm birth with abnormal levels of routinely collected first- and second-trimester biomarkers. Am J Obstet Gynecol 2013;208(06):492.e1-492.e11

5 Jelliffe-Pawlowski LL, Baer RJ, Blumenfeld YJ, et al. Maternal characteristics and mid-pregnancy serum biomarkers as risk factors for subtypes of preterm birth. BJOG 2015;122(11): 1484-1493

6 Jelliffe-Pawlowski LL, Baer RJ, Currier RJ. Second trimester serum predictors of preterm birth in a population-based sample of lowrisk pregnancies. Prenat Diagn 2010;30(08):727-733

7 Olsen RN, Dunsmoor-Su R, Capurro D, McMahon K, Gravett MG. Correlation between spontaneous preterm birth and mid-trimester maternal serum estriol. J Matern Fetal Neonatal Med 2014;27 (04):376-380

8 Blumenfeld YJ, Baer RJ, Druzin ML, et al. Association between maternal characteristics, abnormal serum aneuploidy analytes, and placental abruption. Am J Obstet Gynecol 2014;211(02):144. e1-144.e9

9 McPherson E, Thomas GD, Manlick C, et al. Extreme values of maternal serum analytes in second trimester screening: looking beyond trisomy and NTD's. J Genet Couns 2011;20(04):396-403

10 Walton DL, Norem CT, Schoen EJ, Ray GT, Colby CJ. Secondtrimester serum chorionic gonadotropin concentrations and complications and outcome of pregnancy. N Engl J Med 1999; 341(27):2033-2038

11 Lepage N, Chitayat D, Kingdom J, Huang T. Association between second-trimester isolated high maternal serum maternal serum human chorionic gonadotropin levels and obstetric complications in singleton and twin pregnancies. Am J Obstet Gynecol 2003;188 (05):1354-1359

12 Crandall BF, Robinson L, Grau P. Risks associated with an elevated maternal serum alpha-fetoprotein level. Am J Obstet Gynecol 1991;165(03):581-586

13 Wenstrom KD, Owen J, Davis RO, Brumfield CG. Prognostic significance of unexplained elevated amniotic fluid alpha-fetoprotein. Obstet Gynecol 1996;87(02):213-216
14 Salafia CM, Silberman L, Herrera NE, Mahoney MJ. Placental pathology at term associated with elevated midtrimester maternal serum $\alpha$-fetoprotein concentration. Am J Obstet Gynecol 1988;158(05):1064-1066

15 Dugoff L, Hobbins JC, Malone FDFASTER Trial Research Consortium, et al. Quad screen as a predictor of adverse pregnancy outcome. Obstet Gynecol 2005;106(02):260-267

16 Alleman BW, Smith AR, Byers HM, et al. A proposed method to predict preterm birth using clinical data, standard maternal serum screening, and cholesterol. Am J Obstet Gynecol 2013; 208(06):472.e1-472.e11

17 Sharony R, Zipper O, Amichay K, et al. The magnitude of elevated maternal serum human chorionic gonadotropin and pregnancy complications. J Obstet Gynaecol 2017;37(05):576-579

18 Talge NM, Mudd LM, Sikorskii A, Basso O. United States birth weight reference corrected for implausible gestational age estimates. Pediatrics 2014;133(05):844-853

19 Baer RJ, Norton ME, Shaw GM, et al. Risk of selected structural abnormalities in infants after increased nuchal translucency measurement. Am J Obstet Gynecol 2014;211(06):675.e1-675. e19

20 Kazerouni NN, Currier B, Malm L, et al. . Triple-marker prenatal screening program for chromosomal defects. Obstet Gynecol 2009;114(01):50-58

21 Kazerouni NN, Currier B, Malm L, et al. Triple-marker prenatal screening program for chromosomal defects. Reproduct Endocrinol 2014;16:84-90

22 Baer RJ, Flessel MC, Jelliffe-Pawlowski LL, et al. Detection rates for aneuploidy by first-trimester and sequential screening. Obstet Gynecol 2015;126(04):753-759

23 Norton ME, Baer RJ, Wapner RJ, Kuppermann M, Jelliffe-Pawlowski LL, Currier RJ. Cell-free DNA vs sequential screening for the detection of fetal chromosomal abnormalities. Am J Obstet Gynecol 2016;214(06):727.e1-727.e6

24 Currier R, Wu N, Van Meter K, Goldman S, Lorey F, Flessel M. Integrated and first trimester prenatal screening in California: program implementation and patient choice for follow-up services. Prenat Diagn 2012;32(11):1077-1083

25 Lieppman RE, Williams MA, Cheng EY, et al. An association between elevated levels of human chorionic gonadotropin in the midtrimester and adverse pregnancy outcome. Am J Obstet Gynecol 1993;168(6 Pt 1):1852-1856, discussion 1856-1857 


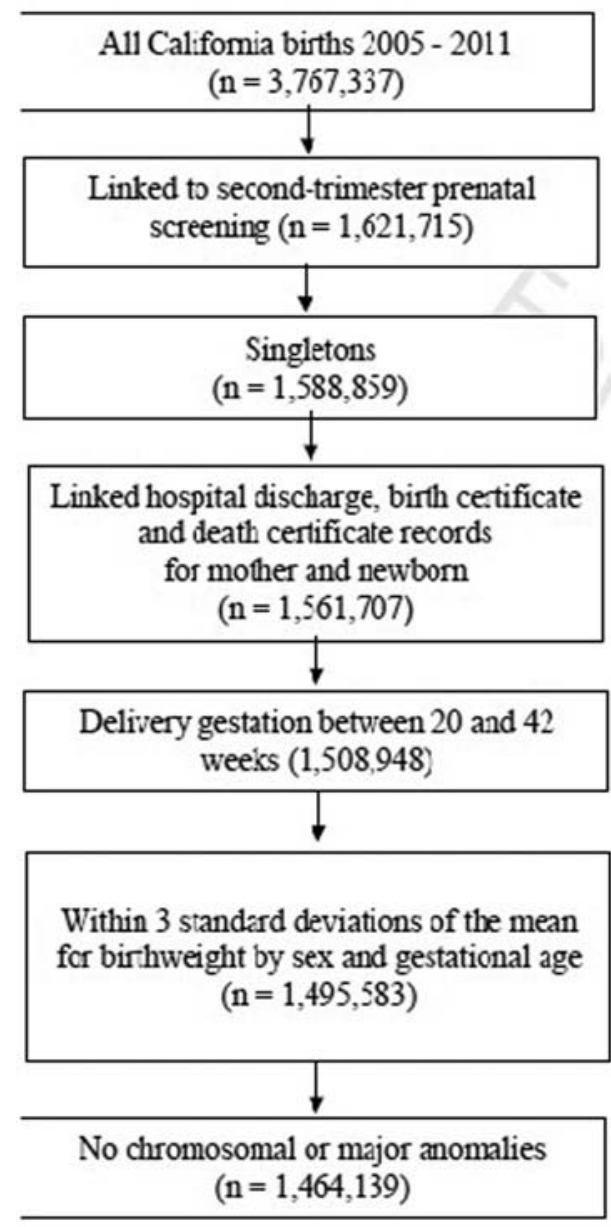

Appendix 1 Sample selection. 